\title{
The Efficacy and Safety of Ablative Fractional Resurfacing Using a 2,940-Nm Er:YAG Laser for Traumatic Scars in the Early Posttraumatic Period
}

\author{
Sun Goo Kim", Eun Yeon Kim², Yu Jin Kim², Se Il Lee ${ }^{2}$ \\ ${ }^{1}$ Seran Plastic Surgery Clinic; ${ }^{2}$ Department of Plastic and Recontructive Surgery, Gachon University Gil Hospital, Incheon, Korea
}

Background Skin injuries, such as lacerations due to trauma, are relatively common, and patients are very concerned about the resulting scars. Recently, the use of ablative and nonablative lasers based on the fractional approach has been used to treat scars. In this study, the authors demonstrated the efficacy and safety of ablative fractional resurfacing (AFR) for traumatic scars using a 2,940-nm erbium: yttrium-aluminum-garnet (Er:YAG) laser for traumatic scars after primary repair during the early posttraumatic period.

Methods Twelve patients with fifteen scars were enrolled. All had a history of facial laceration and primary repair by suturing on the day of trauma. Laser therapy was initiated at least 4 weeks after the primary repair. Each patient was treated four times at 1-month intervals with a fractional ablative 2,940-nm Er:YAG laser using the same parameters. Posttreatment evaluations were performed 1 month after the fourth treatment session.

Results All 12 patients completed the study. After ablative fractional laser treatment, all treated portions of the scars showed improvements, as demonstrated by the Vancouver Scar Scale and the overall cosmetic scale as evaluated by 10 independent physicians, 10 independent non-physicians, and the patients themselves.

Conclusions This study shows that ablative fractional Er:YAG laser treatment of scars reduces scars fairly according to both objective results and patient satisfaction rates. The authors suggest that early scar treatment using AFR can be one adjuvant scar management method for improving the quality of life of patients with traumatic scars.

Keywords Laser therapy / Erbium YAG lasers / Wounds and injuries

\author{
Correspondence: Yu Jin Kim \\ Department of Plastic and \\ Recontructive Surgery, Gachon \\ University Gil Hospital, \\ Namdongdae-ro 774 beon-gil 21, \\ Namdong-gu, Incheon 405-760, \\ Korea \\ Tel: +82-1577-2299 \\ Fax: +82-32-462-2774 \\ E-mail: pseugene@gilhospital.com
}

No potential conflict of interest relevant to this article was reported.

\section{INTRODUCTION}

Skin injuries, such as lacerations or abrasions, due to trauma are relatively common, and patients are very concerned about scars caused by trauma and primary repair [1]. Multiple modalities for improving the clinical appearance of scars have been attempted with varying success, including corticosteroids, dermabrasion, surgical revision, chemical peeling, silicone gel application, pressure therapy, and radiation. Lasers such as the carbon dioxide $\left(\mathrm{CO}_{2}\right)$, erbium: yttrium-aluminum-garnet (Er:YAG), and pulsed dye (PDL) lasers have all been used with differing success in the treatment of scars [2].

Ablative lasers used for skin resurfacing, such as the $\mathrm{CO}_{2}$ and Er:YAG laser, can reduce various scars, but significant adverse 
effects limit their use, and patient downtime can be extensive. Owing to these potential risks, nonablative lasers have been developed as a safe alternative to ablative laser resurfacing, and have been reported to be effective and safe for scars. However, outcomes have remained unsatisfactory and require several treatments to achieve satisfactory efficacy $[1,3]$.

Recently, the use of ablative and non-ablative lasers based on the fractional approach has become a novel strategy for the treatment of scars, and some authors have suggested that treatment with fractional lasers for various scars, such as postoperative, atrophic, and acne scars, has been demonstrated to safely improve the appearance of the scars [1]. However, few studies have been conducted on the efficacy of fractional laser treatment of traumatic scars during the early posttraumatic period.

In this study, we investigated the efficacy and safety of ablative fractional resurfacing (AFR) using a 2,940-nm Er:YAG laser on traumatic scars after primary repair during the early posttraumatic period.

\section{METHODS}

Twelve patients (fifteen scars) of Fitzpatrick skin types III to $V$ were enrolled. All had a history of laceration to the face and were repaired primarily by suturing on the day of trauma. Various types and colors of scars were presented; specific scar characteristics are described in Table 1 . The mean age was 28 years, from 18 to 57 years. Patients with photosensitivity, pregnancy, active lactation, immunosuppression, keloid scarring, current anticoagulant treatment, isotretinoin usage, or ablative resurfacing within the previous year were excluded. Patients signed informed consent forms and granted permission for clinical photographs to be taken. Each patient was treated four times at 1-month intervals using the same parameters, and post-treatment following evaluations was performed 1 month later after the fourth treatment session. Laser therapy was started after at least 4 weeks of the primary repair. To relieve patient discomfort, a 5\% lidocaine cream (EMLA, AstraZeneca, London, UK) with 20 minutes of occlusion, was applied before the treatment sessions, and faces were cleansed with $0.2 \%$ chlorohexidine gluconate. All patients were treated with a fractional ablative 2,940nm Er:YAG laser (LOTUSII, Laseroptek, Sungnam, Korea) at a setting of $1.29 \mathrm{~J} / \mathrm{cm}^{2}$ and a spot diameter of $7 \mathrm{~mm}$. Two laser passes of $400 \mathrm{~mJ}$ in short pulse mode (pulse duration $0.35 \mathrm{~ms}$ ) and one pass of $800 \mathrm{~mJ}$ in long pulse mode (pulse duration 1 $\mathrm{ms}$ ) were performed on scars during each treatment session.

Clinical photographs were taken at baseline and 1 month after the final treatment. Figs. 1-4 show 4 cases of comparative pre- and post-treatment clinical photographs. Three types of assessment methods were used. First, 10 independent physicians scored the scars using the Vancouver Scar Scale (VSS) (Table 2). Second, evaluations at baseline and 1 month after final termination were conducted using pairs of comparative photographs by 10 independent physicians and 10 independent non-physicians using a 10-point scale, to assess clinical improvements in global cosmesis (using a 10-point scale; score 10, excellent improvement, score 1, poor improvement; a higher rating signifies a greater improvement). In addition, patient satisfaction was rated using a 4-point scale (grade 4: > 75\%, excellent improvement; grade 3: $51 \%$ to $75 \%$, good improvement; grade $2: 26 \%$ to $50 \%$, fair improvement; grade 1: $0 \%$ to $25 \%$, poor improvement). Information on side effects and patient satisfaction were collected at each follow-up visit. Statistical analysis of comparisons between the pre- and post-treatment global cosmesis were performed with a Wilcoxon signed-rank test. SPSS ver. 17.0 statistical soft-

\section{Table 1. Assessment of scar characteristics}

\begin{tabular}{|ccclll|}
\hline No. & Age/Sex & Intervals $^{\text {a) }}$ (day) & Cause of laceration & \multicolumn{1}{c|}{ Scar location } & \multicolumn{1}{c|}{ Scar characteristics } \\
\hline 1 & $32 / \mathrm{M}$ & 56 & In-car traffic accident & Forehead & Erythematous, slightly depressed \\
2 & $21 / \mathrm{M}$ & 52 & Pedestrian traffic accident & Forehead, nose & Erythematous, atrophic (forehead), hypertrophic (nose) \\
3 & $22 / \mathrm{F}$ & 34 & Pedestrian traffic accident & Forehead & Erythematous, slightly hypertrophic \\
4 & $27 / \mathrm{F}$ & 38 & Slip and fall & Nose & Erythematous, slightly hypertrophic \\
5 & $57 / \mathrm{M}$ & 30 & Slip and fall & Glabella, eyebrow & Erythematous, slightly depressed \\
6 & $30 / \mathrm{M}$ & 41 & In-car traffic accident & Forehead & Erythematous, flat \\
7 & $30 / \mathrm{M}$ & 41 & In-car traffic accident & Upper eyelid, bilateral & Erythematous, flat \\
8 & $30 / \mathrm{M}$ & 41 & In-car traffic accident & Cheek & Erythematous, flat (perioral), hypertrophic (cheek) \\
9 & $18 / \mathrm{M}$ & 60 & Motorcycle traffic accident & Forehead & Erythematous, flat with focal hypertrophic \\
10 & $19 / \mathrm{F}$ & 52 & In-car traffic accident & Cheek, right chin, philtrum & Erythematous, hypertrophic (philtrum), flat (cheek) \\
11 & $20 / \mathrm{M}$ & 34 & In-car traffic accident & Cheek, bilateral & Erythematous, slightly hypertrophic \\
12 & $20 / \mathrm{M}$ & 34 & In-car traffic accident & Eyelid, bilateral & Erythematous, flat \\
13 & $28 / F$ & 61 & Stab injury by glass & Glabella & Erythematous, flat \\
14 & $41 / M$ & 38 & Stab injury by aluminium & Glabella & Erythematous, hypertrophic \\
15 & $25 / F$ & 74 & In-car traffic accident & Forehead & Erythematous, slightly depressed \\
\hline a) The intervals between the day of trauma and the initiation of laser therapy. & & \\
\hline
\end{tabular}




\section{Fig. 1. Case 1}

(A) A 30-year-old male with facial multiple laceration scars due to a traffic accident. The time interval from trauma to the initiation of laser therapy was 41 days. (B) One month after the conclusion of 4 sessions of laser therapy (Vancouver Scar Scale improvement grade after laser treatment, +4.5 on average by 10 independent physicians; Likert 10-point scale by 10 physicians, 8.1; by non-physicians, 8.0).
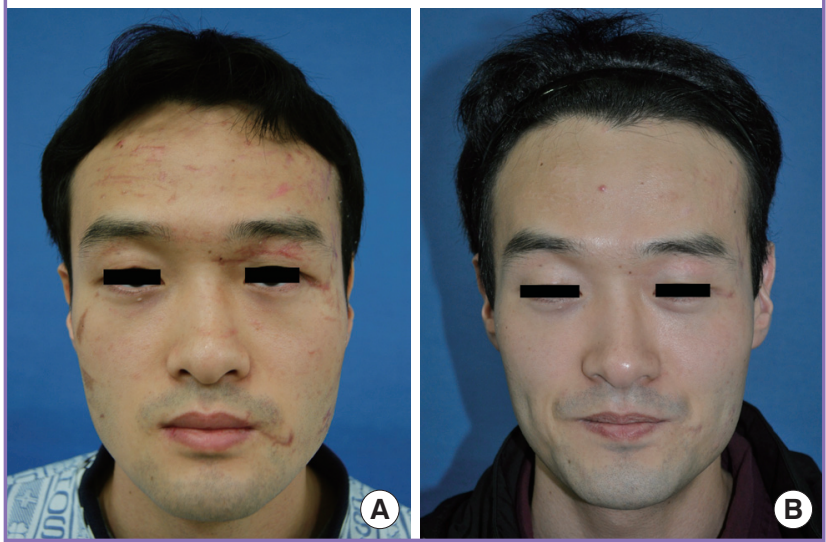

\section{Fig. 2. Case 2}

(A) A 19-year-old female with multiple laceration scars on her right cheek due to a traffic accident. The time interval from trauma to the initiation of laser therapy was 52 days. (B) One month after the conclusion of 4 sessions of laser therapy. Traumatic tattoo on the right. Cheek was treated with two separate applications of ND-YAG laser therapy (Vancouver Scar Scale improvement grade after laser treatment, +3.1 on average by 10 independent physicians; Likert 10-point scale by 10 physicians, 7.5; by non-physicians, 7.3).
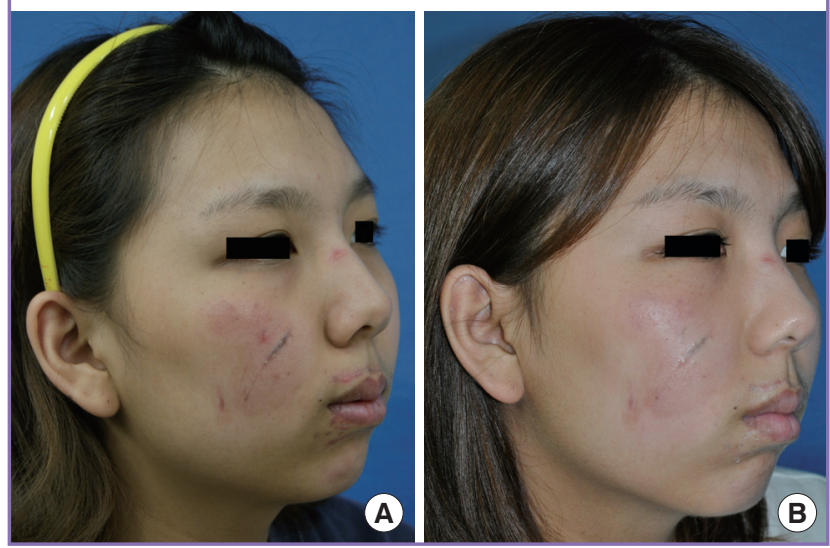

ware (SPSS Inc., Chicago, IL, USA) was used. This study was approved by the Institutional Review Board of Gachon University Gil Hospital (GIRB 0004-2012).

\section{RESULTS}

All 12 patients completed the study. The average time between the day of trauma and the initiation of laser therapy was 45.7 days. At the conclusion of the four times of treatments with the ablative fractional Er:YAG laser, all treated portions of the scars

\section{Fig. 3. Case 3}

(A) A 18-year-old male with multiple facial laceration scars due to a motorcycle accident. The time interval from trauma to the initiation of laser therapy was 60 days. (B) One month after the conclusion of 4 sessions of laser therapy (Vancouver Scar Scale improvement grade after laser treatment: +2.5 on average by 10 independent physicians; Likert 10-point scale by 10 physicians, 7.0; by non-physicians, 7.1).
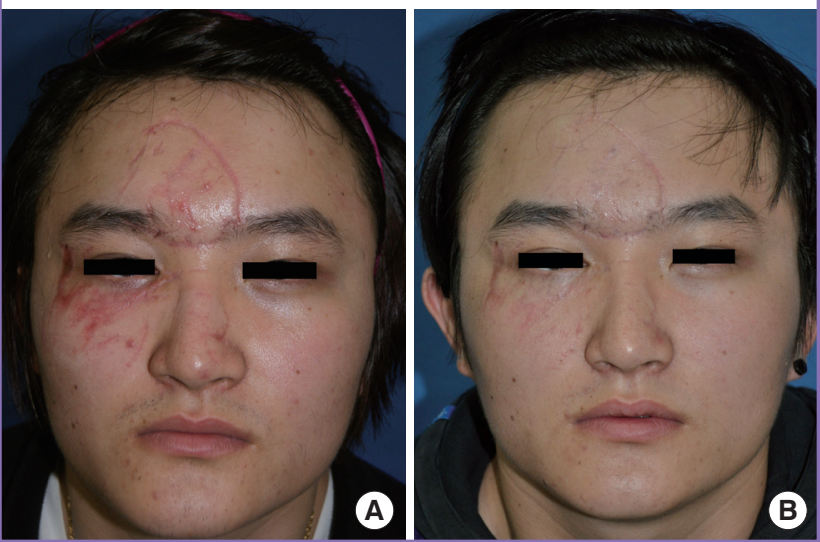

\section{Fig. 4. Case 4}

(A) A 22-year-old female with forehead laceration scars due to a traffic accident. The time interval from trauma to the initiation of laser therapy was 34 days. (B) One month after the termination of 4 sessions of laser therapy (Vancouver Scar Scale improvement grade after laser treatment, +4.7 on average by 10 independent physicians; Likert 10-point scale by 10 physicians, 7.3; by non-physicians, 9.1).
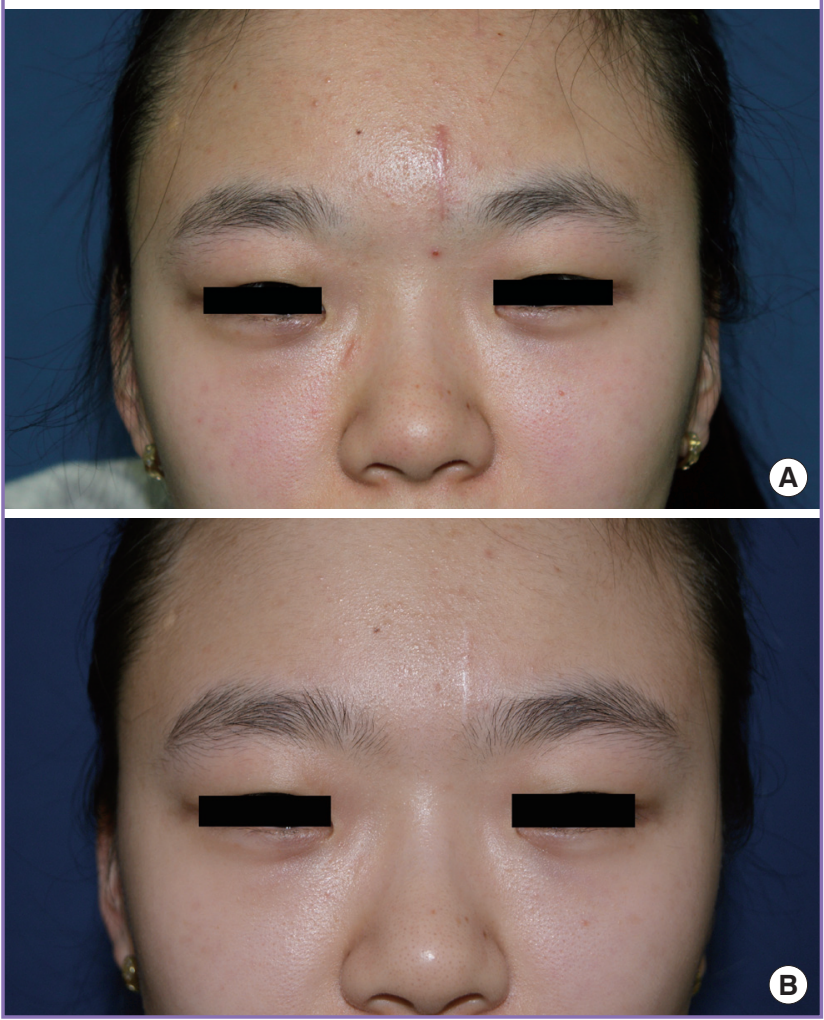

showed improvements, as demonstrated by the VSS and the overall cosmetic scale evaluated by 10 blinded physicians, 10 


\section{Table 2. Vancouver Scar Scale}

\begin{tabular}{cl}
\hline Pigmentation & Normal color (resembles nearby skin) \\
0 & Hypopigmentation \\
1 & Hyperpigmentation \\
2 & Normal \\
Vascularity & Pink (slightly increased in local blood supply) \\
0 & Red (significant increase in the local blood supply) \\
1 & Purple (excessive local blood supply) \\
2 & \\
3 & Normal \\
Pliability & Supple (flexible with minimal resistance) \\
0 & Yielding (giving way to pressure) \\
1 & Firm (solid/inflexible, not easily moved, resistant to manual pressure) \\
2 & Banding (rope-like, blanches with extension of scar, does not limit range of motion) \\
3 & Contracture (permanent shortening of scar producing deformity or distortion; limits range of motion) \\
4 & \\
5 & Normal (flat) \\
Height $(\mathrm{mm})$ & $<2$ \\
0 & $>2$ and $<5$ \\
1 & $>5$ \\
2 &
\end{tabular}

Table 3. Scar assessment before and after 4 sessions of fractional laser treatment

\begin{tabular}{|cccc|}
\hline \multirow{2}{*}{ No. } & \multicolumn{2}{c}{ Vancouver Scar Scale score } & \multirow{2}{*}{$\begin{array}{c}\text { Improvement } \\
\text { grade }\end{array}$} \\
\cline { 2 - 3 } & Pretreatment & Posttreatment & \\
\hline 1 & 6.7 & 0.5 & 6.2 \\
2 & 6.5 & 5.1 & 1.4 \\
3 & 6.0 & 1.3 & 4.7 \\
4 & 5.8 & 3.4 & 2.4 \\
5 & 7.6 & 2.7 & 4.9 \\
6 & 4.9 & 0.3 & 4.6 \\
7 & 6.3 & 1.7 & 4.6 \\
8 & 4.1 & 0.9 & 3.2 \\
9 & 6.3 & 3.8 & 2.5 \\
10 & 5.3 & 2.6 & 2.7 \\
11 & 8.6 & 6.7 & 1.9 \\
12 & 3.2 & 2.3 & 0.9 \\
13 & 2.6 & 1.4 & 1.2 \\
14 & 4.9 & 2.5 & 2.4 \\
15 & 3.9 & 1.6 & 2.3 \\
Mean & 5.5 & 2.4 & 3.1 \\
\hline The numbers represent the average of 10 independent physicians. \\
\hline
\end{tabular}

blinded non-physicians, and the patients themselves. The mean VSS scores were 5.5 before treatment and 2.4 at 1 month after final treatment. After laser treatment, the mean VSS values decreased significantly $(\mathrm{P}<0.05)$ (Table 3$)$. The follow-up results obtained by the 10 independent physicians and by the 10 independent non-physicians at 1 month after final treatment showed marked improvement that was evaluated with a Likert 10-point scale (Fig. 5). The mean grade of clinical improvement based on assessments of the clinical photographs by the 10 independent physicians was 7.0 and those by the 10 independent non-physicians was 6.9. In addition, $13.3 \%$ of the patients graded improve-

\section{Fig. 5. Follow-up results by physicians and non-physicians}

Follow-up results obtained by the 10 blinded physicians and 10 blinded non-physicians at 1 month after final treatment showed marked improvement as evaluated with a Likert 10-point scale. The mean grade by the physicians was 7.0 , and 6.9 by the non-physicians.

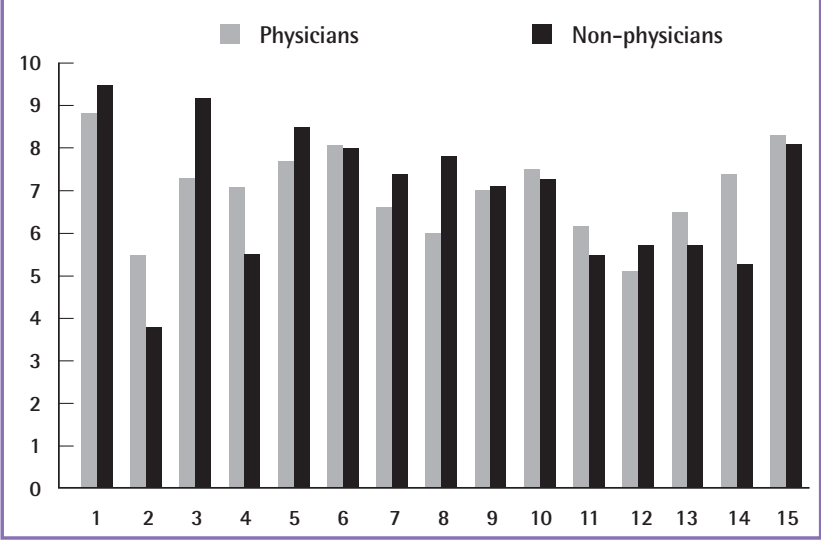

Table 4. Evaluation of improvement after ablative fractional laser by patient satisfaction

\begin{tabular}{|lc|}
\hline Improvement & Patient satisfaction \\
\hline Grade 4, excellent $(\geq 75 \%)$ & $2(13.3)$ \\
Grade 3, good $(50 \%-75 \%)$ & $12(80.0)$ \\
Grade 2, fair $(25 \%-50 \%)$ & $1(6.7)$ \\
Grade 1, poor ( $\leq 25 \%)$ & $0(0.0)$ \\
\hline $\begin{array}{l}\text { Values are presented as number (\%). } \\
\text { The mean grade of clinical improvement based on patients' own satisfaction was } \\
3.07 .\end{array}$ \\
\hline
\end{tabular}

ments as near total ( $>75 \%$ ), $80.0 \%$ as marked ( $51 \%$ to $75 \%$ ), and $6.7 \%$ as moderate improvements (26\% to $50 \%$ ) (Table 4 ). The mean grade of clinical improvement based on patients' own 
satisfaction was 3.07.

\section{DISCUSSION}

The treatment of various scars with lasers is a relatively new concept that is gaining in popularity. Over the past decade, laser skin resurfacing with $\mathrm{CO}_{2}$ and Er:YAG lasers has become a popular choice for the treatment of atrophic scars. However, although highly effective at re-contouring the skin and improving scar appearance, treatment with these ablative lasers has been associated with extended recovery periods, prolonged erythema, and other untoward side effects. Because of these potential risks, nonablative technology using long-pulsed infrared (1,450-nm diode and 1,320-nm neodymium:yttrium-aluminum-garnet) laser systems was developed as a safer alternative to ablative technology for creation of a controlled thermal injury to the dermis with subsequent neocollagenesis and remodeling of scarred skin [4]. Due to a need for more noticeable clinical improvement than these latter nonablative systems could provide, fractional photothermolysis was most recently introduced into the skin resurfacing market [5].

Fractional photothermolysis (FP) produces arrays of microscopic thermal wounds called microscopic treatment zones (MTZs) at specific depths in the skin without injuring the surrounding tissues. Because uninjured tissue surrounds each MTZ, the intact dermis rapidly heals around these columns of thermal damage, which in turn stimulates progressive collagen remodeling [6]. Nonablative FP originally used near-infrared energy, which spared the epidermis, but the more aggressive concept of ablative FP, in which the MTZs include the epidermis, has been established using Er:YAG and $\mathrm{CO}_{2}$ lasers [7].

The mechanism by which a laser affects scar remodeling is unknown, but AFR may influence the secretion of various cytokines and growth factors by stimulating a variety of as yet unelucidated cellular responses and blood vessels [1]. Some authors have reported that while they can only conjecture why fractional photothermolysis yields such positive responses in scarred skin, it is possible that the controlled, limited dermal heating initiates a cascade of events in which normalization of the collagenesis/ collagenolysis cycle occurs [5].

AFR has been reported to be a safe and effective method for treating various scars as well as facial rhytides and photodamaged skin [1].

Previous studies performed with the first generation of fractionated Er:YAG laser (Fraxel SR750, Reliant Technologies Inc., Mountain View, CA, USA) approved for use at $40 \mathrm{~mJ}$ by the US Food and Drug Administration in 2003, have reported marked improvement in $90 \%$ of patients with mild to moderate atrophic acne scars [5]. Narurkar [8] reported that, for acne scars, fractional laser treatment provides more consistent results than any other treatment modality; it is also appropriate for the treatment of patients with darker skin.

Hu et al. [9] demonstrated the successf ul treatment of atrophic facial acne scars using the two modes of ablation and coagulation of fractionally delivered Er:YAG (2,940-nm) laser with acceptable downtime and minimal side-effects. Using the fractional $\mathrm{CO}_{2}$ laser, similar results have been noted. Chapas et al. [10] discovered a mean $66.8 \%$ improvement in 13 patients with facial atrophic acne scarring within two to three treatment sessions.

In a case study, a patient with a surgical scar on the chin was treated with $1,550 \mathrm{~nm}$ fractional photothermolysis and the authors suggested that fractional photothermolysis has the potential to be effective and safe in treating surgical scars [11]. Choe et al. [12] demonstrated that a fractional laser can successfully suppress formation of hypertrophic scarring in total thyroidectomy patients. Jung et al. [1] also reported the safety and efficacy of treatment with ablative $\mathrm{CO}_{2}$ fractional laser for thyroidectomy scarring in the early postoperative period.

In the present study, VSS scores for traumatic scars were significantly lower after laser treatment. Our objective results showed that ablative fractional Er:YAG laser treatment improved scar appearance fairly well without clinically prolonged erythema or hyperpigmentation. Furthermore, this study had a $93.3 \%$ satisfaction rate based on an excellent or good rating of patient satisfaction. However, the improvements for widened and/or hypertrophic scars after AFR were relatively low, and thus, we suggest that scar revision and adjuvant therapy with steroid injection could be considered before AFR for the improvement of widened and/or hypertrophic scars.

Although we did not compare the treatment group with a control group, early treatment using AFR may prevent scar formation and aid rapid scar improvement. Further histologic studies are needed to assess whether AFR improves the existing scars or has any preventative abilities; In addition, a split-scar study to compare the effects of laser assisted and natural healing would be informative.

The concept of using lasers as prophylaxis against scarring during the early postoperative and posttraumatic period is relatively new. It has been proposed that treatment with PDL or non-ablative fractional lasers repress scar formation and promote scar improvements during the early postoperative period $[2,12,13]$. However, the appropriate window for laser treatment is unknown [1]. In the present study, we chose patients treated at least 4 weeks after primary repair because we thought that reepithelialization and stabilization of the repaired wound would 
be complete at this time.

In a study by Nouri et al. [13], it was demonstrated that treatment with a non-ablative modality, such as a PDL, may be safely started on the day of suture removal. Furthermore, the early treatment of scars has been reported to minimize many of the undesirable cosmetic results, such as hypertrophy, increased erythema, and hard, contracted scar formation [2]. Choe et al. [12] used a 1,550-nm fractional erbium glass laser for total thyroidectomy patients, who were treated four times at 1-month intervals using a laser starting 2 or 3 weeks after the surgery, which is the time a surgical wound presumably completes its epithelization and stitches are removed.

One could argue that the scars treated in the present study would have improved on their own because scars undergo remodeling for up to 6 or even 12 months. The optimum timing of laser treatment and the mechanism underlying the effects of early laser treatment have not been determined. Further studies should be conducted to evaluate the potential prophylactic effects of laser treatment against postoperative scarring and the underlying mechanisms [1].

In this study, we used the VSS to provide a more objective measurement of scarring.

Although several scar evaluation tools have been developed, no tool has been universally accepted. Factors contributing to scar formation include the following: pigmentation, vascularity, thickness (scar height), pliability, surface texture, and surface area. The VSS scales used in the present study used four values (pigmentation, pliability, height, and vascularity) to rate scarring. Adverse effects and complications related to fractional laser treatments were evaluated at all follow-up visits. Common adverse effects resulting from laser irradiation, such as the reactivation of herpes labialis, postinflammatory hyperpigmentation, dyspigmentation, and the outbreak of acneiform eruptions, did not occur in the present study.

In conclusion, the present study shows that ablative fractional Er:YAG laser treatment improved scars fairly well based on objective results and patient satisfaction rates. Even though AFR cannot be the only method for scar management, we believe that early scar treatment using AFR can be an adjuvant scar management method for improving the quality of life of patients with traumatic scars. Finally, we suggest that a long term study with a larger number of patients should be conducted to compare scars treated with AFR and those that are not.

\section{REFERENCES}

1. Jung JY, Jeong JJ, Roh HJ, et al. Early postoperative treatment of thyroidectomy scars using a fractional carbon dioxide laser. Dermatol Surg 2011;37:217-23.

2. Conologue TD, Norwood C. Treatment of surgical scars with the cryogen-cooled $595 \mathrm{~nm}$ pulsed dye laser starting on the day of suture removal. Dermatol Surg 2006;32:13-20.

3. Park GH, Rhee DY, Bak H, et al. Treatment of atrophic scars with fractional photothermolysis: short-term follow-up. J Dermatolog Treat 2011;22:43-8.

4. Tanzi EL, Alster TS. Comparison of a $1450-\mathrm{nm}$ diode laser and a 1320-nm Nd:YAG laser in the treatment of atrophic facial scars: a prospective clinical and histologic study. Dermatol Surg 2004;30:152-7.

5. Alster TS, Tanzi EL, Lazarus M. The use of fractional laser photothermolysis for the treatment of atrophic scars. Dermatol Surg 2007;33:295-9.

6. Manstein D, Herron GS, Sink RK, et al. Fractional photothermolysis: a new concept for cutaneous remodeling using microscopic patterns of thermal injury. Lasers Surg Med 2004;34:426-38.

7. Hantash BM, Bedi VP, Chan KF, et al. Ex vivo histological characterization of a novel ablative fractional resurfacing device. Lasers Surg Med 2007;39:87-95.

8. Narurkar VA. Skin rejuvenation with microthermal fractional photothermolysis. Dermatol Ther 2007;20 Suppl 1:S10-3.

9. $\mathrm{Hu} \mathrm{S}, \mathrm{Hsiao}$ WC, Chen MC, et al. Ablative fractional erbium-doped yttrium aluminum garnet laser with coagulation mode for the treatment of atrophic acne scars in Asian skin. Dermatol Surg 2011;37:939-44.

10. Chapas AM, Brightman L, Sukal S, et al. Successful treatment of acneiform scarring with $\mathrm{CO} 2$ ablative fractional resurfacing. Lasers Surg Med 2008;40:381-6.

11. Behroozan DS, Goldberg LH, Dai T, et al. Fractional photothermolysis for the treatment of surgical scars: a case report. J Cosmet Laser Ther 2006;8:35-8.

12. Choe JH, Park YL, Kim BJ, et al. Prevention of thyroidectomy scar using a new 1,550-nm fractional erbium-glass laser. Dermatol Surg 2009;35:1199-205.

13. Nouri K, Jimenez GP, Harrison-Balestra C, et al. 585-nm pulsed dye laser in the treatment of surgical scars starting on the suture removal day. Dermatol Surg 2003;29:65-73. 